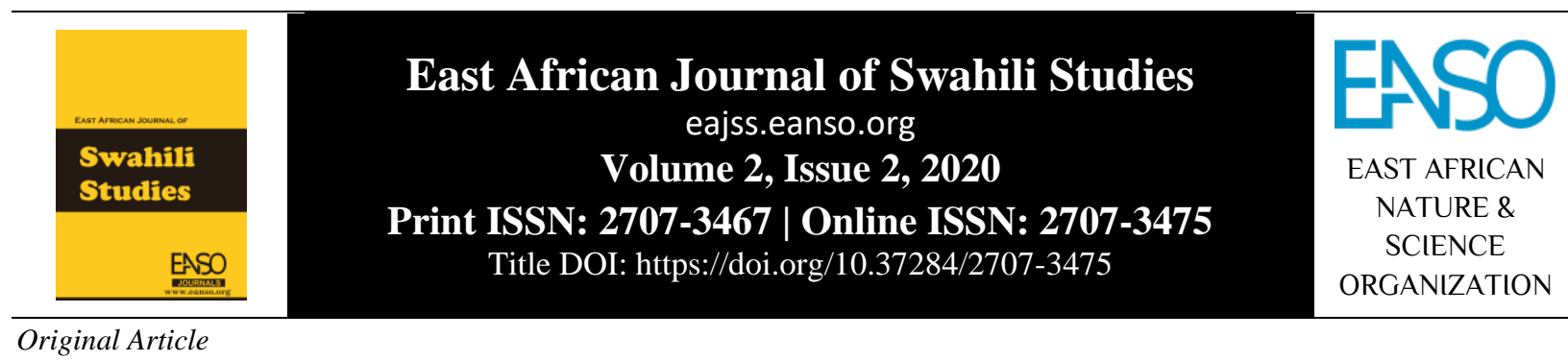

\title{
Visababishi vya Idadi Kubwa ya Wanafunzi wa Shule za Sekondari Nchini Uganda Kutochukua Somo la Kiswahili
}

\author{
Dr. Willy Wanyenya ${ }^{*}$ \\ ${ }^{1}$ Makerere University Business School, P. O. Box 1337, Kampala, Uganda. \\ *Barua pepe ya mawasiliano: willywanyenya@gmail.com \\ ORCID: https://orcid.org/0000-0002-7594-8256
}

DOI ya Nakala: https://doi.org/10.37284/eajss.2.2.159

Tarehe ya Uchapishaji: IKISIRI

05 June 2020 Katika makala haya, mtafiti anaeleza visababishi vya idadi kubwa ya wanafunzi katika shule za upili nchini Uganda kutochukua somo la Kiswahili.

Maneno Muhimu: Wakati wa kupunguza idadi ya masomo kwa kila mwanafunzi unapofika,

wanafunzi huwa na sababu za kimsingi zinazowafanya wapendelee masomo

Mwelekeo Hasi,

Lugha Ya Kigeni,

Mhemko,

Mtazamo.

fulani na kuyakataa mengine. Kwa kawaida wanafunzi huwa hawalazimishwi

kuchukua masomo fulani. Kila mwanafunzi huwa na uhuru wa kuchagua

masomo anayoyapenda. Imebainika wazi kuwa nchini Uganda, wakati wa

wanafunzi kupunguza idadi ya masomo unapofika, wengi wao huwa

wanaacha somo la Kiswahili. Katika darasa la wanafunzi mia moja, ni wanafunzi kumi au kumi na watano tu ndio wanachukua Kiswahili. Hali hii inatisha sana hasa kwa wapenzi wa Kiswahili. Hivyo basi, mtafiti aliendeleza utafiti huu ili kueleza sababu zinazopelekea wanafunzi kufanya hivyo. Dhamira ya utafiti ni kueleza bayana kwa nini wanafunzi wengi nchini Uganda huwa hawachukui somo la Kiswahili. Madhumuni ya utafiti huu ni kueleza mielekeo ya wanafunzi wa shule za sekondari nchini Uganda dhidi ya ujifunzaji wa lugha ya Kiswahili na kufafanua hatua ambazo zinastahili kuchukuliwa ili kufanya idadi ya wanafunzi wa Kiswahili iongezeke. Katika kazi hii, mkabala wa utafiti ulikuwa wa nyanjani. Kwa kutumia mkabala huu, mtafiti alizuru nyanjani na kuendeleza mahojiano na wahojiwa wake. Utafiti huu umeonyesha kuwa wanafunzi wa shule za sekondari nchini Uganda huwa hawapendelei somo la Kiswahili kwa sababu zifuatazo: kuwepo na sera mbovu nchini kuhusu lugha ya Kiswahili; wanafunzi kutokuwa na msingi wa lugha ya Kiswahili; wanafunzi kuwa na fikra potovu kuwa Kiswahili ni lugha ya wezi; wanafunzi kufikiria kuwa Kiswahili ni somo linalofaa wenzao kutoka Kenya na Tanzania; wanafunzi kufikiria kuwa lugha ya Kiswahili ni ngumu; 
watu wa nchi ya Uganda kuchukulia Kiswahili kuwa lugha ya kawaida na isiyofaa kufundishwa shuleni; wananchi wa Uganda kupendelea Kiganda na Kiingereza na kupiga vita lugha ya Kiswahili.

\section{APA CITATION}

Wanyenya, W. (2020). Visababishi vya Idadi Kubwa ya Wanafunzi wa Shule za Sekondari Nchini Uganda Kutochukua Somo la Kiswahili. East African Journal of Swahili Studies, 2(2), 1-10. https://doi.org/10.37284/eajss.2.2.159

\section{CHICAGO CITATION}

Wanyenya, Willy. 2020. "Visababishi vya Idadi Kubwa ya Wanafunzi wa Shule za Sekondari Nchini Uganda Kutochukua Somo la Kiswahili”. East African Journal of Swahili Studies 2 (2), 1-10. https://doi.org/10.37284/eajss.2.2.159.

\section{HARVARD CITATION}

Wanyenya, W. (2020) "Visababishi vya Idadi Kubwa ya Wanafunzi wa Shule za Sekondari Nchini Uganda Kutochukua Somo la Kiswahili”, East African Journal of Swahili Studies, 2(2), pp. 1-10. doi: 10.37284/eajss.2.2.159.

\section{IEEE CITATION}

W. Wanyenya, "Visababishi vya Idadi Kubwa ya Wanafunzi wa Shule za Sekondari Nchini Uganda Kutochukua Somo la Kiswahili”, EAJSS, vol. 2, no. 2, pp. 1-10, June 2020.

\section{MLA CITATION}

Wanyenya, Willy. "Visababishi vya Idadi Kubwa ya Wanafunzi wa Shule za Sekondari Nchini Uganda Kutochukua Somo la Kiswahili”. East African Journal of Swahili Studies, Vol. 2, no. 2, June 2020, pp. 1-10, doi:10.37284/eajss.2.2.159.

\section{UTANGULIZI}

Kwa vile nchi zetu zimeungana pamoja na kuunda jumuiya ya Afrika Mashariki, lugha ya Kiswahili inastahili kufundishwa shuleni katika nchi wanachama. Hii ni kwa sababu Kiswahili ndiyo lugha ya mawasiliano katika eneo hili la Afrika Mashariki. Watu kutoka nchi ya Uganda huwa wanaenda Kenya au Tanzania kufanya biashara au kufanya kazi zinginezo. Watu kutoka nchi hizo vile vile huwa wanazuru nchi ya Uganda kufanya vivyo hivyo. Na ikizingatiwa kuwa lugha ya Kiswahili ndiyo inazungumzwa na watu wengi sana katika eneo hili la Afrika Mashariki kuliko lugha zingine, bila shaka, inastahili kukuzwa. Lakini ni jambo la kusikitisha kuona wanafunzi wengi nchini Uganda hawataki kusoma lugha hii. Wao huwa wanasoma Kiswahili katika kidato cha 1 na cha 2 kwa sababu ni somo la lazima kwao. Kama si kuwa somo la lazima, basi, wengi wao wasingelichukua miongoni mwa masomo yao. Hili ni jambo hatari sana kwao kwa sababu watakosa kuajiriwa kazi ambazo zinahitaji shahada za juu katika Kiswahili. Kwa hiyo, jambo hili linastahili kushughulikiwa mapema na nchi za Afrika Mashariki. Serikali husika zina wajibu wa kuhakikisha kuwa ufundishaji wa lugha ya Kiswahili katika shule za nchi zao unaimarishwa. Serikali ya Uganda inastahili kubuni sera maalumu kuhusu lugha ya
Kiswahili. Sera hiyo itapelekea lugha hii kuimarishwa kote nchini. Kutokana na sera hiyo, juhudi mbalimbali zitafanywa ili kukuza na kuendeleza lugha hii. Serikali ikibuni sera hiyo, bila shaka, wanafunzi wataona umuhimu wa kujifunza lugha ya Kiswahili. Jambo hili litapelekea idadi ya wanafunzi wa Kiswahili kuongezeka.

\section{SUALA LA UTAFITI}

Kulingana na mtafiti, Kiswahili kimefanywa kuwa somo la lazima kwa wanafunzi wa shule za sekondari kuanzia kidato cha 1 hadi cha 2 nchini Uganda. Katika kidato cha tatu, wanafunzi wana uhuru wa kupunguza idadi ya masomo kwa kuacha baadhi yao na kubakisha machache ya kuendelea nayo hadi kidato cha 4 . Katika kidato cha 1 na cha 2, wanafunzi wote huwa wanafanya Kiswahili. Kwa kawaida katika shule moja, idadi yao inaweza kuwa 100 au zaidi, kulingana na wanafunzi wanaosajiliwa. Hata hivyo, wakifika katika kidato cha 3, wengi wao huacha Kiswahili. Katika kidato cha tatu, idadi ya wanafunzi wa Kiswahili huwa inapungua hadi wanafunzi 10 au 15 . Katika baadhi ya shule, idadi ya wanafunzi wa Kiswahili hupungua hadi wanafunzi 5. Idadi ya wanafunzi wa Kiswahili kupungua kiasi hiki ni jambo lililohitaji kutafitiwa. Kwa hiyo, ni jambo hili ndilo lililompelekea mtafiti kuendeleza utafiti huu akiwa 
na lengo la kueleza kisa kinachopelekea wanafunzi kutochukua somo la Kiswahili kila wanapojiunga na kidato cha 3. Katika kazi hii, dhamira ya utafiti ni kueleza bayana kwa nini wanafunzi wengi kwenye shule za sekondari nchini Uganda hawataki kuendelea na somo la Kiswahili hadi kidato cha 4. Hivyo basi, madhumuni ya utafiti ni kueleza mielekeo ya wanafunzi wa shule za upili dhidi ya ujifunzaji wa lugha ya Kiswahili nchini uganda na kufafanua hatua ambazo zinastahili kuchukuliwa ili kufanya idadi ya wanafunzi wa Kiswahili iongezeke katika shule za sekondari nchini uganda.

\section{MAPITIO YA MAANDISHI}

Kwa mujibu wa Kovac na Zdilar (2017), tafiti nyingi zimefanywa kuhusu mielekeo, imani na mihemko ya wanafunzi wanaojifunza lugha za kigeni. Kulingana na mtaalamu huyu, mielekeo ya wanafunzi ni mojawapo ya mambo yanayopelekea wanafunzi kufaulu katika ujifunzaji lugha. Kovac na Zdilar wanasema jambo hili lilitambuliwa na walimu wanaofundisha lugha za kigeni na limethibitishwa na wasomi kadhaa katika tafiti zao. Kovac na Zdilar (2017) waliwanukuu Victori na Lockhart (1995) wanaponena kuwa mtazamo na imani ya wanafunzi kuhusu lugha za kigeni kwa jumla huzingatia imani zao wenyewe. Hivyo, mitazamo hii mbalimbali huathiri ujifunzaji lugha na aina ya ujifunzaji lugha. Horwitz (1987) anadai kuwa kuelewa imani za wanafunzi kuhusu lugha fulani husaidia walimu kufahamu wanafunzi wao vizuri. Kwa mfano, walimu wanapata kufahamu matarajio ya wanafunzi, umakinifu wao na mafanikio yao. Hii ni kwa sababu baada ya walimu kuwafahamu wanafunzi wao, wanaweza kuondoa mielekeo hasi wanayoishikilia kuhusu lugha. Hali hii hufanikisha ujifunzaji lugha kwa wanafunzi. Tukizingatia maelezo haya tunaona kuwa mwandishi huyu amezungumzia mambo mbalimbali yanayoathiri ujifunzaji wa lugha ya kigeni. Hata hivyo, ni wazi kwamba mwandishi hajatoa maelezo kuhusu mambo yanayopelekea wanafunzi wa lugha ya kigeni kuwa na mwelekeo endelezi au mwelekeo hasi. Jambo hili linaacha mwanya ambao mtafiti ameuziba katika kazi hii.

Karahan (2007) anasisitiza dhima ya mielekeo ya wanafunzi dhidi ya ujifunzaji wa lugha ya kigeni. Anaeleza kuwa wanafunzi wanaojifunza lugha ya kigeni wanastahili kuelekezwa kiutaratibu na walimu wao ili kuondoa mielekeo hasi inayowapelekea kuchukia lugha ya kigeni. Anasema kuwa hii ni kwa sababu ni jukumu la walimu kufanya wanafunzi wao wafurahie kujifunza lugha ya kigeni badala ya kuichukia. Choy na Troudi (2006) wanasema kuwa hisia na mihemko ya wanafunzi wanaojifunza lugha ya kigeni huathiri mitazamo na fikra zao kuhusu lugha wanayojifunza. Karahan (2007) anaendelea kusema kuwa wanafunzi kuwa na kiwango cha juu cha mhemko na hamu ya kujifunza lugha ya kigeni huleta matokeo mazuri. Wataalamu hawa wanasema kuwa mihemko inayodhihirishwa na wanafunzi wanaojifunza lugha ya kigeni ni jambo la kimsingi linaloleta ufanisi haraka sana katika ujifunzaji lugha. Tukitazama maelezo haya tunaona kuwa mwandishi ametaja wajibu wa walimu wa kuwaelekeza wanafunzi kiutaratibu ili wapate kufurahia kujifunza lugha ya kigeni. Hii inamaanisha kuwa hata wanafunzi wakiwa na mielekeo hasi dhidi ya lugha ya kigeni, walimu wana uwezo wa kuwafanya wafurahie kujifunza lugha hiyo. Kwa hiyo, ni jambo la kushangaza kuona kwamba licha ya kuwa na walimu wa Kiswahili, idadi kubwa ya wanafunzi nchini Uganda inaacha Kiswahili. Jambo hili vile vile linaacha mwanya ambao mtafiti ameuziba katika kazi hii.

Smith (1971) anasema kuwa wasomi wengi wa makala yake wanaweza kuambatisha neno mwelekeo na wanafunzi. Hii ni kwa sababu mwelekeo ndio unaofanya mambo kuwa magumu au rahisi kwa mwanafunzi anapokuwa anajifunza lugha darasani. Mwanafunzi akiwa na mwelekeo endelezi dhidi ya lugha ya kigeni anayojifunza, mambo humwia rahisi na akiwa na mwelekeo hasi, basi, mambo humwia magumu. Kwa mujibu wa mtaalamu huyu, watu huwa wanajifunza mambo vile vile wanaweza kukosa pia kujifunza. Hali kadhalika, kwa vile watu huwa wanajifunza mambo, basi, mambo hayo yanaweza kufundishwa. Kwa hiyo, kupenda au kutopenda lugha ya kigeni ni mambo ya kujifunza. Hii inamaanisha kuwa kupenda lugha ya kigeni ni jambo la kujifunza na kutopenda lugha hiyo vile vile ni jambo la kujifunza. Hakuna mtu anayezaliwa akiwa anapenda lugha au kuichukia. Mtoto akiingia darasani bila kuwa na mwelekeo hasi dhidi ya lugha ya kigeni anayotarajiwa kufundishwa, basi, matokeo yake yatakuwa mazuri na atafanikiwa 
katika kujifunza lugha hiyo ya kigeni. Katika maelezo haya, mwandishi anasema kupenda na kuchukia lugha fulani ni mambo ya kujifunza. Vile vile, mwandishi huyu anasema kuwa kwa sababu watu huwa wanajifunza mambo, wao wanaweza kukosa kujifunza. Hata hivyo, hajaeleza nini kinachowafanya washindwe kujifunza. Hii ina maana kuwa nchini Uganda kuna mambo yasiyojulikana ambayo yanafanya wanafunzi kuchukia lugha ya Kiswahili badala ya kuipenda lugha hiyo. Bila shaka, jambo hili vile vile linaacha mwanya ambao mtafiti ameuziba katika kazi hii.

Tukirejelea maelezo ya Mingyong (2015), mwelekeo ni jambo muhimu sana katika ujifunzaji wa lugha ya kigeni. Anasema jambo hili limezingatiwa sana na watafiti kadha wa kadha. Hata hivyo, mtaalamu huyu anaendelea kusema kuwa wasomi wengi hawajashughulikia dhima ya mwelekeo katika ujifunzaji wa lugha ya kigeni. Kulingana na utafiti wa Mingyong (2015), mwelekeo wa mwanafunzi dhidi ya lugha ya kigeni husababishwa na mielekeo ya wazazi, uhusiano baina ya mwalimu na mwanafunzi na uhusiano baina ya mwanafunzi na wanafunzi wenzake. Mingyong anasisitiza kuwa mambo haya yanaweza kumpelekea mwanafunzi kupenda na kufurahia kujifunza lugha ya kigeni au kuichukia. Taarifa hii inaonyesha kuwa wazazi wanaweza kufanya watoto wao wachukie lugha fulani au wanaweza kuwafanya waipende lugha hiyo. Wazazi wanaweza kufanya hivyo kwa kuongea mambo mazuri au mabaya kuhusu lugha ya kigeni. Wakiongea mambo mazuri kuhusu lugha hiyo ya kigeni, bila shaka watoto wao wataipenda. Na wakiongea mambo mabaya kuihusu, basi, watoto wao wataichukia. Vile vile, uhusiano mbaya baina ya mwanafunzi na mwalimu wake unaweza kufanya mwanafunzi aichukie lugha au aipende. Hii ni kwa sababu kuna baadhi ya walimu ambao hufanya wanafunzi wachukie somo lao badala ya kuwaelekeza kiutaratibu na kuwafanya walipende. Hali kadhalika, mwanafunzi anaweza kuichukia lugha ya kigeni kutokana na mambo anayoyasikia kutoka kwa wanafunzi wenzake. Kwa mfano akiwasikia wanasema lugha fulani haina maana kwao, basi, mwanafunzi pia ataichukia lugha hiyo bila kuwa na uhakika. Nchini Uganda, tatizo halijulikani liko wapi. Hatujui tatizo limetokana na wazazi, walimu au na wanafunzi kwa jumla. Bila shaka, jambo hili pia linaacha mwanya ambao umezibwa katika kazi hii.

Kwa mujibu wa Oroujlou na Vahedi (2011), mhemko, mielekeo na imani kuhusu ujifunzaji lugha ni miongoni mwa mambo yanayoweza kufanikisha juhudi za wanafunzi wanaojifunza lugha ya kigeni. Kwa hiyo, walimu wanastahili kuzingatia mambo haya kwa makini. Wakiwanukuu Latchanna na Dagnew (2009), Oroujlou na Vahedi (2015) wanasema kuwa mwelekeo umekubaliwa kama dhana muhimu katika kufahamu tabia za binadamu. Mwelekeo umefasiliwa kama hali ya kiakili inayojumlisha fikra, imani na hisia. Wataalamu hawa wanasema kuwa imani kuhusu lugha anayojifunza mtu inaambatishwa moja kwa moja na mafanikio yake darasani. Imani za wanafunzi kuhusu lugha zinaweza kuwa kizuizi kwao wakiamini kwamba hawawezi kujifunza lugha husika. Fikra hasi kama hizi na kutokuwa na mhemko huwa vizuizi katika ujifunzaji lugha ya kigeni. Lakini fikra hasi za wanafunzi zinaweza kubadilishwa na wakafanikiwa katika kujifunza lugha ya kigeni. Mwanafunzi kuwa na fikra endelezi dhidi ya lugha ni msingi mzuri wa kuanza kujifunza lugha hiyo. Tukirejelea maelezo haya, tunaona kwamba Oroujlou na Vahedi (2015) wamezungumzia mchango wa fikra hasi na fikra endelezi katika kujifunza lugha ya kigeni. Hata hivyo, hajaeleza bayana fikra hasi ni zipi na fikra endelezi ni gani. Kwa hiyo, jambo hili vile vile linaacha mwanya ambao mtafiti ameuziba katika utafiti huu.

Kulingana na Ahmed (2015), watu wanaohusika na ufundishaji wa lugha husema kwamba wanafunzi wenye nia ya kujifunza lugha bila shaka hufanikiwa licha ya mazingira wanamosomea. Mtaalamu huyu anasema kuwa mhemko unaoletwa darasani na wanafunzi ni jambo muhimu linalowaletea ufanisi katika juhudi zao za kujifunza lugha ya kigeni. Mhemko ni msukumo ilio ndani ya mwanafunzi humpa motisha ya kuendelea kujaribu kupata kile anachokitaka. Kwa ujumla, wanafunzi wenye mhemko na wenye malengo maalumu ni rahisi kufundishwa kuliko wenzao ambao hawana. Hii inamaanisha kuwa wanafunzi wenye nia ya kujifunza lugha watajitahidi kuona wanaishika lugha wanayojifunza katika hali yoyote ile. Wanafunzi kama hawa hawatakubali jambo lolote kuwazuia kujifunza lugha ya kigeni. Hata lugha 
yenyewe ikiwa ngumu kiasi gani, wanafunzi hao watang'ang'ana nayo mpaka waweze kufaulu. Kutokana na maelezo haya, inabainika wazi kuwa kila mtu anaweza kujifunza lugha ya kigeni akiwa na nia ya kufanya hivyo. Ni jambo la kushangaza kuona kwamba nchini Uganda wanafunzi hawang'ang'ani kujifunza lugha ya Kiswahili. Swali ni je, wanafunzi hawana nia ya kujifunza lugha? Swali hili, bila shaka linaacha mwanya ambao mtafiti ameuziba katika kazi hii.

Kwa mujibu wa Habók na Magyar (2018), katika miaka ya hivi karibuni, wasomi wameendeleza tafiti nyingi kuhusu ujifunzaji wa lugha za kigeni kwa kusisitiza mikakati inayotumiwa na watu wanaojifunza lugha hizo. Tafiti kadha wa kadha zimethibitisha kuwa mikakati hiyo hufanya wanafunzi wawe chonjo darasani na kukuza umilisi wa lugha lengwa baada ya kuhitimu shule. Wataalamu hawa wanasema kuwa licha ya tafiti nyingi kufanywa, kuna maelezo machache sana yanayodhihirisha uhusiano baina ya mikakati ya kujifunza lugha na mielekeo ya wanafunzi dhidi ya lugha ya kigeni, alama ya lugha hiyo na matokeo ya jumla shuleni. Kauli hii inaonyesha kwamba kuna mahitaji ya kuendeleza utafiti wa ziada kuhusu ujifunzaji wa lugha na kubainisha uhusiano baina ya mikakati ya kujifunza lugha na mielekeo ya wanafunzi dhidi ya lugha hizo.

Rahimi na Yadollahi (2011) wanadai kuwa kutokana na upeo mpana wa matumizi ya teknolojia ya tarakilishi katika mafunzo, mambo mengi yanayohusiana na matumizi ya tarakilishi yameangaziwa zaidi katika kipindi cha mwongo mmoja uliopita. Wasomi sasa wanajitahidi kueleza uhusiano baina ya umilisi wa lugha na utumiaji wa tarakilishi kwa ajili ya masomo. Kwa mujibu wa wataalamu hawa, lugha huwa inafanikisha mafunzo yanayotekelezwa kwa njia ya tarakilishi. Hata hivyo, wanasema kwamba mambo kama vile mielekeo, mihemko, imani na mitazamo ya watu wanaojifunza lugha ya kigeni yanastahili kuzingatiwa. Tunaposoma maandishi haya, tunaona mwandishi akisisitiza mambo kama vile mielekeo, mihemko, imani na mitazamo katika ujifunzaji wa lugha ya kigeni. Hata hivyo, mwandishi hajaeleza mambo yanayopelekea wanafunzi kuwa na mielekeo na imani tofauti kuhusu lugha za kigeni, jambo ambalo mtafiti amelishughulikia katika kazi hii.
Kwa mujibu wa Abdelkader na Amine 2017), mwelekeo huchangia pakubwa sana ujifunzaji wa lugha ya kigeni. Hii ni kwa sababu wanafunzi wenye mielekeo endelezi huwa wanazuia athari za kushindwa katika juhudi zao za kujifunza lugha. Wao hawaruhusu mielekeo hasi kutawala uwezo wao kujifunza lugha ya kigeni. Hivyo basi, dhana ya mwelekeo ni muhimu sana katika ujifunzaji wa lugha ya kigeni. Kwa hiyo, katika kazi hii, mtafiti ameeleza jinsi hali ya kujifunza lugha ya Kiswahili ilivyo nchini Uganda.

Hosseini na Pourmandnia (2013) wanasema ili aelewa wanafunzi wanaojifunza lugha ya kigeni kwa kina, mwalimu anastahili kujifunza imani zao kuhusu lugha anayowafunza. Kwa mujibu wa mtaalamu huyu, wanafunzi wanaojifunza lugha ya kigeni wanapoingia darasani huwa wanaleta mambo mbalimbali ya kibinafsi yakiwemo mielekeo, imani na mitindo ya kilugha katika mazingira wanamosomea. Kwa mujibu wa wataalamu hawa, karibu wasomi wote wanakubali mafanikio katika ujifunzaji lugha ya kigeni huchangiwa kwa kiasi kikubwa na fikra za wanafunzi wenyewe, jinsi wanavyoichukulia lugha hiyo, watu wanaoizungumza, utamaduni wa wazungumzaji pamoja na mazingira wanamosomea. Mambo haya yote yana mchango mkubwa sana katika ujifunzaji wa lugha ya kigeni. Mtaalamu huyu anasema kuwa ingawa jambo la kutafiti mielekeo na imani za wanafunzi haliwezi kuleta mafanikio katika ujifunzaji wa lugha ya kigeni, kwa upande mwingine jambo hilo huwa kama mwongozo jinsi wanafunzi walivyo muhimu katika mazingira wanamosomea. Hii ni kwa sababu kabla ya mwalimu kuanza kufundisha ni jambo la busara kufahamu mielekeo na imani za wanafunzi kuhusu lugha anayotaka kuwafunza. Kwa kufanya hivyo, mwalimu akitambua kuwa wanafunzi wake wana mielekeo hasi dhidi ya lugha anayotaka kuwafunza, basi, anaweza kuona namna ya kuwaelekeza na kubadilisha fikra hizo hasi na kuwafanya wafurahie somo lake. Mambo haya yalimpelekea mtafiti kuendeleza utafiti huu ili aeleze jinsi hali ilivyo nchini Uganda.

Kulingana na Abu-Snoubar (2017) kama anavyowanukuu Abidin, Mohammadi na Alzwari (2012), kwa jumla mwelekeo ni mtazamo wa binadamu dhidi ya hali au kitu fulani kulingana na fikra au imani zake. Al Noursi (2013) na 
Eshghinejad (2016) wanaeleza mwelekeo kama mchakato wa kisaikolojia ambao huchangia wanafunzi hupenda au huchukia hali fulani. Kulingana na wataalamu hawa, ujifunzaji wa lugha ya kigeni hauwezi kutenganishwa na mielekeo ya wanafunzi. Hii ni kwa sababu mielekeo endelezi huchangia kwa kuwapa wanafunzi motisha ya kujifunza lugha ilhali mielekeo hasi huwa kizuizi. Taarifa hii inaonyesha umuhimu wa mielekeo ya wanafunzi katika kujifunza lugha za kigeni. Hata hivyo, mwandishi hajaeleza mielekeo ya wanafunzi dhidi ya lugha za kigeni katika nchi mbalimbali. Jambo hili linaacha mwanya ambao umezibwa na mtafiti katika kazi hii.

Kwa mujibu wa Al-Musnad (2018), lugha ni kiini cha utamaduni na urithi wa nchi yoyote ile. Lugha hudhihirisha mawazo na mienendo ya binadamu. Mtu anapojifunza lugha ya kigeni huwa hajifunzi lugha hiyo tu bali huwa anajifunza pia desturi na utamaduni, mambo ambayo yana uhusiano na lugha hiyo hiyo. Kulingana na mtaalamu huyu, mtu akijifunza lugha, huwa anashika desturi na utamaduni wa lugha hiyo. Kwa hiyo, tamaduni na desturi za wazungumzaji wa lugha zinaweza kuwa vizuizi kwa watu wanaotaka kujifunza lugha hizo. Hata hivyo, haijafahamika kama mambo haya yameathiri wanafunzi wanaojifunza lugha ya Kiswahili nchini Uganda. Jambo hili pia linaacha mwanya ambao mtafiti ameuziba katika kazi hii.

Kwa mujibu wa Fatiha na wenzake (2014), wakati wa kujifunza lugha, wanafunzi hutarajiwa kuingia darasani wakiwa na mielekeo ya kujifunza lugha au kuwa na mielekeo hasi. Wanafunzi huwa na mielekeo tofauti kuhusu darasa wanamosomea, mwalimu anayewafundisha pamoja na mtaala unaofuatwa. Hii ni kwa sababu wanafunzi wanaweza kutopenda darasa wanamosomea, wanaweza kuwa na chuki dhidi ya mwalimu wao wa lugha na wanaweza kuchukia mtaala unaofuatwa katika kufundisha lugha. Mambo haya yote yanaweza kuathiri masomo ya wanafunzi. Kulingana na Fatiha na wenzake (2014), mambo makuu yanayoathiri wanafunzi wanaojifunza lugha ni mielekeo na mihemko. Mambo haya mawili yana athari endelezi na athari hasi kwa wanafunzi. Kwa mujibu wa wataalamu hawa, wanafunzi wakiwa na mielekeo endelezi hufanikiwa katika masomo yao. Lakini wakiwa na mielekeo hasi, wao huwa hawafanikiwi. Hata hivyo, mwandishi hajaeleza mambo yanayopelekea wanafunzi kuwa na mielekeo tofauti, masuala ambayo mtafiti ameyashughulikia katika kazi hii.

Nilay (2018) anaeleza kuwa kuna mambo makuu sita yanayoathiri mihemko katika ujifunzaji wa lugha za kigeni. Anasema mambo haya ni mielekeo kuhusu lugha lengwa; imani za mtu anayejifunza lugha hiyo; malengo ya kujifunza lugha hiyo; kushiriki, mazingira wezeshi na uzoevu wa mtu anayejifunza lugha. Kulingana na mtaalamu huyu, mielekeo huanza kukua mtoto akiwa mdogo na huathiriwa na umri wake, wazazi, watoto wenzake, mwingiliano na watu wanaotofautiana kiutamaduni na kijamii, watu wanaotofautiana kulingana na hali za kiuchumi pamoja na mazingira wanamoishi. Kwa hiyo, mambo haya sita yana athari kubwa sana katika ujifunzaji wa lugha za kigeni. Hata hivyo, haijajulikana kama masuala haya sita ndiyo yamepelekea wanafunzi wa shule za sekondari nchini Uganda kukataa kuendelea kusoma lugha ya Kiswahili. Jambo hili vile vile linaacha mwanya ambao mtafiti ameuziba katika kazi hii.

\section{Hoja Kuu ambazo Zimejitokeza katika Maandiko Mbalimbali}

Jedwali 1: Hoja Kuu Ambazo Zimejitokeza Kuhusu Ujifunzaji wa Lugha ya Kigeni

\begin{tabular}{ll}
\hline Na & Hoja \\
\hline 1 & $\begin{array}{l}\text { Mwelekeo endelezi hufanikisha ujifunzaji } \\
\text { wa lugha ya kigeni }\end{array}$ \\
2 & $\begin{array}{l}\text { Mwelekeo hasi huzuia ujifunzaji wa lugha } \\
\text { ya kigeni }\end{array}$ \\
3 & $\begin{array}{l}\text { Mwelekeo hasi unaweza kubadilishwa na } \\
\text { walimu wanaofunza lugha ya kigeni }\end{array}$ \\
4 & $\begin{array}{l}\text { Imani endelezi hufanikisha ujifunzaji wa } \\
\text { lugha ya kigeni }\end{array}$ \\
5 & $\begin{array}{l}\text { Imani hasi huzuia ujifunzaji wa lugha ya } \\
\text { kigeni }\end{array}$ \\
6 & $\begin{array}{l}\text { Mhemko vile vile hufanikisha ujifunzaji wa } \\
\text { lugha ya kigeni }\end{array}$ \\
7 & $\begin{array}{l}\text { Ukosefu wa mhemko huzuia ujifunzaji wa } \\
\text { lugha ya kigeni }\end{array}$ \\
\hline
\end{tabular}

Tukiangalia Jedwali 1, tunaona kuwa mwelekeo endelezi hufanikisha ujifunzaji wa lugha ya kigeni. Mwelekeo endelezi kwa mujibu wa utafiti huu ni hali ya mwanafunzi kuwa na fikra nzuri kuhusu lugha anayojifunza. Hoja hii inamaanisha kuwa 
mwanafunzi akiwa na fikra nzuri kuhusu lugha ya kigeni, huwa akifanikiwa katika kujifunza lugha hiyo. Ni jambo la kawaida kuwa mwanafunzi yeyote akiingia darasani akiwa na moyo wa kujifunza lugha ya kigeni huwa chonjo akitaka kushika maelezo ya mwalimu wake. Mwanafunzi wa aina hii hushika lugha ya kigeni haraka sana.

Jedwali 1 vile vile linaonyesha kuwa mwelekeo hasi huzuia ujifunzaji wa lugha ya kigeni. Mwelekeo hasi kwa mujibu wa utafiti huu ni hali ya mwanafunzi kuingia darasani akiwa na chuki dhidi ya lugha ya kigeni anayojifunza. Hoja hii inamaanisha kuwa mwanafunzi akiingia darasani akiwa na chuki dhidi ya lugha anayojifunza, huwa hafanikiwi. Mwanafunzi wa aina hii hukumbana na hali ngumu darasani. Kwa kawaida, mambo huwa magumu kwake na hawezi kushika lugha ya kigeni anayojifunza.

Kwa kurejelea Jedwali 1, tunaona pia kwamba mwelekeo hasi unaweza kubadilishwa na mwalimu anayefundisha lugha ya kigeni. Mwalimu akifanya hivyo, basi, hata wanafunzi wenye mwelekeo hasi wanaweza kufaulu katika kujifunza lugha hiyo ya kigeni. Hoja hii inamaanisha kuwa mwalimu ana wajibu wa kuona kwamba wanafunzi wake wote wanafaulu katika kujifunza lugha ya kigeni anayoifundisha. Asipofanya hivyo, basi, inamaanisha kuwa mwenyewe ana tatizo.

Tukitazama jedwali la 1, inabainika wazi kuwa imani endelezi vile vile hufanikisha ujifunzaji wa lugha ya kigeni. Ni kweli kwamba wanafunzi huwa na imani tofauti kuhusu masomo yao. Baadhi yao hufikiria kuwa masomo fulani yana maana kwao kuliko masomo mengine. Kwa hiyo, imani kama hizi huathiri pakubwa sana matokeo yao. Wanafunzi wanaochukulia somo Fulani kuwa la maana kwao hufaulu katika juhudi zao za kujifunza lugha.

Jedwali 1 vile vile linaonyesha kuwa imani hasi za wanafunzi dhidi ya lugha ya kigeni huzuia ujifunzaji wa lugha hiyo. Hoja hii inamaanisha kuwa wanafunzi wanaoingia darasani wakiwa na imani hasi dhidi ya lugha wanayojifunza huwa hawafanikiwi. Hii ni kwa sababu baadhi ya wanafunzi huingia darasani wakiwa na mawazo mabaya dhidi ya lugha wanayojifunza. Wanafunzi kama hawa wasipoelekezwa vizuri na mwalimu wao hawawezi kufanikiwa katika kujifunza lugha.

Hali kadhalika, Jedwali 1 linadhihirisha kuwa wanafunzi kuwa na mhemko hufanikisha ujifunzaji wa lugha ya kigeni. Kwa mujibu wa utafiti huu, mhemko ni hali ya wanafunzi kuchangamkia somo fulani darasani. Wao huwa wanataka wafundishwe somo hilo. Wakikosa kufundishwa wao huwa wanakasirika. Wakifundishwa huwa wanafurahi. Mwalimu akichelewa kuigia darasani, wanafunzi humtafuta. Wanafunzi hao hufanya mazoezi ya kutamka maneno, kuandika maneno, kutaja vitenzi, kutaja nomino na kutunga sentensi mbalimbali. Wanafunzi wa aina hii hufanikiwa katika juhudi zao za kujifunza lugha hiyo.

Tukizingatia Jedwali 1, tunaona kwamba ukosefu mihemko huzuia ujifunzaji wa lugha ya kigeni. Wanafunzi wanaoingia darasani bila mhemko huwa hawafanikiwi. Hii ni kwa sababu wanafunzi hao huwa hawataki kuhudhuria vipindi vya lugha hiyo, mwalimu akichelewa kuingia darasani hawamtafuti, mwalimu akikosa wanafurahi na hawafanyi mazoezi kama wenzao wenye mhemko. Kwa hiyo, wanafunzi wa aina hii huwa hawafanikiwi katika juhudi zao za kujifunza lugha. Hali kadhalika, wanafunzi huwa wakitoka shuleni kama hawana umilisi wa lugha hiyo ya kigeni.

\section{MATOKEO YA UTAFITI}

Kwa mujibu wa Jedwali 2, idadi kubwa ya wanafunzi huacha kusoma Kiswahili nchini Uganda hutokana na sera mbovu ya lugha. Jambo hili linathibitishwa na idadi kubwa ya wahojiwa $100(100 \%)$ ambao walikubali hoja hii. Kulingana nao, nchini Uganda, sera inaruhusu wanafunzi kufundishwa Kiswahili katika kidato cha 1 na cha 2. Kufanya mambo kuwa magumu zaidi, Kiswahili hakifundishwi katika shule za msingi. Wanafunzi huwa wakilazimishwa kusoma lugha hii wanapojiunga na shule za sekondari. Kwa hiyo, wengi wao huwa wakikumbana na mambo ambayo ni mapya na magumu kwao. Hali hii hupelekea wanafunzi wengi kuacha Kiswahili baada ya kumaliza kidato cha pili. 
Jedwali 2: Mara Tokezi ya Visababishi Vya Wanafunzi Kuacha Lugha ya Kiswahili

\begin{tabular}{lll}
\hline Hoja & Mara Tokezi & Asilia \\
\hline Kuwa na sera mbovu nchini kuhusu lugha ya Kiswahili & 100 & $100 \%$ \\
Wanafunzi kutokuwa na msingi wa lugha ya Kiswahili & 100 & $100 \%$ \\
Wanafunzi kuwa na fikra potovu kuwa Kiswahili ni lugha ya wezi & 68 & $68 \%$ \\
Wanafunzi kufikiria kuwa Kiswahili ni somo linalofaa wenzao kutoka Kenya & 88 & $88 \%$ \\
na Tanzania & & 74 \\
Wanafunzi kufikiria kuwa lugha ya Kiswahili ni ngumu & $96 \%$ \\
Jumuiya kuchukulia Kiswahili kuwa lugha ya kawaida na isiyofaa & 96 & $100 \%$ \\
kufundishwa shuleni \\
$\begin{array}{l}\text { Wananchi kupendelea Kiganda na Kiingereza na kupiga vita lugha ya } \\
\text { Kiswahili }\end{array}$
\end{tabular}

Tukiangalia Jedwali 2, tunaona vile vile kuwa wanafunzi katika shule za upili nchini Uganda huwa hawataki kuendelea na Kiswahili hadi kidato cha 4 kwa sababu ya kutokuwa na msingi wa lugha hii. Hoja hii vile vile ilidhibitishwa na wahojiwa wote $(100 \%)$ ambao waliikubali. Wahojiwa ambao walikuwa wanafunzi walisema kwamba wao huwa wakikabiliwa na matatizo katika somo la Kiswahili kwa sababu ya kutokuwa na msingi imara. Walisema wao hufundishwa Kiswahili kwa mara ya kwanza wakiwa katika kidato cha 1 . Walisema jambo hili hufanya mambo yawe magumu kwao na kuwalazimu kuaacha Kiswahili.

Wanafunzi katika shule za upili nchini Uganda huwa hawataki kuendelea na somo la Kiswahili kutokana na fikra potovu kuwa Kiswahili ni lugha ya wezi. Hoja hii ilidhibitishwa na wahojiwa 68 (68\%) (Jedwali 2). Fikra hizi bila shaka zimetokana na historia mbaya ya Uganda. Hii ni kwa sababu wakati wa utawala wa Amini, watu waliuawa kwa wingi sana huku wengine wakiibiwa mali zao. Wakati huo, majangili walitumia maneno ya Kiswahili 'fungua mlango.' Kuanzia hapo, wananchi wengi wamekuwa wakisema kuwa Kiswahili ni lugha ya wezi. Mwelekeo huu wa watu wazima umeathiri vijana na kuwafanya wachukie lugha ya Kiswahili.

Wanafunzi wengi nchini Uganda huacha somo la Kiswahili kwa sababu ya fikira kuwa hili ni somo linalofaa wanafunzi kutoka Kenya na Tanzania. Jambo hili vile vile lilidhibitishwa na wahojiwa 88 (88\%) ambao walikubali hoja hiyo (Jedwali 2). Wanafunzi waliohojiwa walisema kuwa wao huwa wakiacha somo Kiswahili kwa sababu hawana msingi imara. Wanafunzi hao hawafikirii kwamba hata bila kuwa na msingi wa Kiswahili wanaweza kufundishwa lugha hii na wakaishika.

Ilidhibitishwa pia kuwa wanafunzi katika shule za sekondari nchini Uganda hukiacha Kiswahili kwa sababu ya kuwa na imani potovu kwamba somo la Kiswahili ni gumu. Jambo hili lilithibitishwa na wahojiwa 74 (74\%) ambao walikubali hoja hii (Jedwali 2). Kutokana na imani hii, wanafunzi wengi huwa wanaogopa kuendelea na somo hili hadi kidato cha 4. Imani hiyo inapelekea wanafunzi kujiunga na shule za sekondari kama wameamua kutoendelea na Kiswahili hadi kidato cha 4. Wanafunzi pia hawataki kuendelea na somo la Kiswahili hadi kidato cha 4 kwa sababu jumuiya inaona Kiswahili kama lugha ya kawaida na isiyofaa kufundishwa shuleni. Jambo hili lilithibitishwa na wahojiwa 96 (96\%) (Jedwali 2). Hali hii imepelekea baadhi ya wazazi kushauri watoto wao wasiendelee na lugha ya Kiswahili. Wanafunzi kama hao wanasubiri tu kumaliza kidato cha 2 halafu waache lugha hii.

Halikadhalika, wanafunzi katika shule za upili nchini Uganda huwa hawataki kuendelea na somo la Kiswahili hadi kidato cha 4 kwa sababu ya wananchi kupendelea lugha zingine na kupiga vita Kiswahili. Ni jambo la wazi kuwa nchini Uganda ufalme wa Buganda unataka lugha yao ambayo ni Kiganda ikuzwe katika eneo lao. Viongozi wa ufalme huo hawataki kuona lugha nyingine ikiendelezwa katika eneo hilo. Kwa hiyo, hawataki kabisa kusikia kwamba Kiswahili kimefanywa kuwa somo la lazima kuanzia shule za msingi hadi kidato cha 4. Wanaona uimarishaji wa Kiswahili ni tishio kubwa sana kwa lugha yao asili. Mbali na hili, wananchi katika maeneo mengine nje ya 
ufalme wa Buganda huwa wanapendelea lugha ya Kiingereza. Badala ya kuhamasisha watoto wao wajifunze lugha ya Kiswahili, wao huwa wakiwahamasisha wajifunze lugha ya Kiingereza. Jambo vile vile lilithibitishwa na wahojiwa 100 (100\%) (Jedwali 2).

\section{HITIMISHO}

Matokeo ya utafiti huu yanaonyesha kuwa kuna sababu za kimsingi ambazo zimeathiri maendeleo ya lugha ya Kiswahili nchini Uganda. Kwanza serikali yenyewe imeshindwa kufanya Kiswahili kuwa somo la lazima katika shule za chekechea, za msingi na za sekondari. Halafu kuna mitazamo potovu ya wananchi kuhusu lugha ya Kiswahili. Mambo haya yameathiri pakubwa sana maendeleo ya Kiswahili nchini Uganda. Hii ni kwa sababu yamepelekea wanafunzi kuwa na mielekeo hasi dhidi ya lugha ya Kiswahili. Kwa hiyo, ili Kiswahili kipate kuendelea katika nchi hii, serikali inastahili kuchukua hatua madhubuti bila kujali kabila lolote lile na kufanya Kiswahili kuwa somo la lazima katika shule zote nchini. Hali kadhalika, tume ya Kiswahili ya Afrika Mashariki inapaswa kuingilia kati na kuendeleza miradi mbalimbali nchini Uganda ili kufanya wananchi waone umuhimu wa Kiswahili. Kwa mfano, tume hii inaweza kufadhili vipindi vya Kiswahili kwa kutumia vituo mbalimbali vya redio kwa ajili ya kuhamasisha jamii kuhusu umuhimu wa lugha hii, kufadhili wanafunzi wanaojiunga na vyuo mbalimbali kwa mafunzo ya ualimu katika Kiswahili na kuhakikisha kuwa watu wanaohitimu katika Kiswahili wanaajiriwa katika nchi yoyote iliyo mwanachama wa jumuiya ya Afrika Mashariki. Mambo haya yakitekelezwa, bila shaka, lugha hii itaheshimiwa kama ilivyo katika nchi za Kenya na Tanzania. Kwa hiyo, suala la Kiswahili nchini Uganda lisiwe la serikali ya nchi hii bali liwe la jumuiya ya Afrika Mashariki. Na si nchi ya Uganda peke yake bali nchi zote za Afrika Mashariki ambazo hazijapiga hatua katika Kiswahili. Tume hii ihakikishe kuwa hatua za kuimarisha Kiswahili zinachukuliwa katika nchi wanachama kama vile Rwanda, Burundi na Sudan ya Kusini, nchi ambazo zimebaki nyuma katika masuala ya Kiswahili.

\section{MAREJELEO}

Abdelkader, M., \& Amine, D. M. (2017). Exploring students' attitudes towards learning English as a foreign language A semi-structured research interviewing with third year LMD students at Saida University. Aleph, 8, 89-109.

Abidin, M, Mohammadi, M. \& Alzwari, H. (2012). EFL Students' Attitudes towards Learning English Language: The Case of Libyan Secondary School Students. Asian Social Science, 8(2), 119-134.

Abu-Snoubar, T. K. (2017). An Evaluation of EFL Students' Attitudes Toward English Language Learning in Terms of Several Variables. International Journal of English Language Teaching, 5(6), 18-34.

Ahmed, S. (2015). Attitudes towards English Language Learning among EFL Learners at UMSKAL. Journal of education and practice, 6(18), 6-16.

Al Noursi, O. (2013). Attitude towards Learning English: The case of the UAE Technological High School. Educational Research, 4(1), 2130 .

Al-Musnad, B. I. (2018). The Role of Motivation and Attitude in Second Language Learning: A study of Arabic Language Learning among Foreign Female Nurses in Riyadh, Saudi Arabia. Journal of Applied Linguistics and Language Research, 5(1), 157-183.

Choy, S., \& Troudi, S. (2006). An investigation into the changes in perceptions of and attitudes towards learning English in a Malaysian college. International Journal of Teaching and Learning in Higher Education, 18(2), 120-130.

Eshghinejad, S. (2016). EFL Students' Attitudes toward Learning English Language: The Case Study of Kashan University Students. Cogent Education, 3(1), 1-13.

Fatiha, M., Sliman, B., Mustapha, B., \& Yahia, M. (2014). Attitudes and motivations in learning English as a foreign language. International Journal of Arts \& Sciences, 7(03), 117-128. 
Habók, A., \& Magyar, A. (2018). The effect of language learning strategies on proficiency, attitudes and school achievement. Frontiers in psychology, 8, 2358.

Horwitz, E. K. (1987). Surveying student beliefs about language learning. In A. Wenden, \& J. Rubin (Eds.), Learner strategies in language learning (pp. 119-129). Englewood Cliffs, NJ: Prentice-Hall.

Hosseini, S. B., \& Pourmandnia, D. (2013). Language learners' attitudes and beliefs: Brief review of the related literature and frameworks. International Journal on New Trends in Education and Their Implications, 4(4), 63-74.

Karahan, F. (2007). Language attitudes of Turkish students towards the English language and its use in Turkish context. Cankaya University Journal of Arts and Sciences, 1(7), 73-87.

Kovac, M. M., \& Zdilar, A. M. (2017). Students' attitudes towards foreign languages. Journal of Education and Developmental Psychology, 7(2), 124-133.

Latchanna, G., \& Dagnew, A. (2009). Attitude of teachers towards the use of active learning methods. E-journal of All India Association for Educational Research, 21(1).

Mingyong, Z. (2015, February). The effect of attitude on foreign language learning and teaching in institutes of higher vocational education based on affective filter hypothesis. In 2015 International Conference on Futuristic Trends on Computational Analysis and Knowledge Management (ABLAZE) (pp. 728733). IEEE.

Nilay, A. V. C. I. (2018). Attitudes of state school students towards learning English as a foreign language. Uluslararasl Güncel Eğitim Araştırmaları Dergisi, 4(1), 41-51.

Oroujlou, N., \& Vahedi, M. (2011). Motivation, attitude, and language learning. ProcediaSocial and Behavioral Sciences, 29, 994-1000.

Rahimi, M., \& Yadollahi, S. (2011). Foreign language learning attitude as a predictor of attitudes towards computer-assisted language learning. Procedia Computer Science, 3, 167174.

Smith, A. N. (1971). The importance of attitude in foreign language learning. The Modern Language Journal, 55(2), 82-88.

Victori, M., \& Lockhart, W. (1995). Enhancing metacognition in self-directed language learning. System, 23, 223-234 